\title{
A 5-step systematic therapy (FSST) for treating plugged ducts and mastitis in breastfeeding women
}

\section{Yuzhi Yao}

Guangzhou Women and Children's Medical Center https://orcid.org/0000-0001-5602-6216

\section{Tianzhu Long}

Guangzhou Women and Children's Medical Center

\section{Yuhong Pan}

Guangzhou Women and Children's Medical Center

\section{Yin Li}

Guangzhou Women and Children's Medical Center

\section{Ling Wu}

Guangzhou Women and Children's Medical Center

\section{Benjie Fu}

Guangzhou Women and Children's Medical Center Hongmin Ma ( $\sim$ hongminmaa@sina.com)

Guangzhou Women and Children's Medical Center https://orcid.org/0000-0003-0464-1203

\section{Research}

Keywords: Breastfeeding, breastfeeding support, massage, plugged ducts, breast pain, mastitis

Posted Date: May 22nd, 2020

DOI: https://doi.org/10.21203/rs.3.rs-29777/v1

License: (c) (1) This work is licensed under a Creative Commons Attribution 4.0 International License.

Read Full License 


\section{A 5-step systematic therapy (FSST) for treating plugged ducts and mastitis in}

breastfeeding women

Yuzhi Yao\#, Tianzhu Long ${ }^{\#}$, Yuhong Pan, Yin Li, Ling Wu, Benjie Fu, Hongmin Ma*

Department of Breast Surgery, Guangzhou Women and Children's Medical Center, Guangzhou 510623, China

*Corresponding author: Hongmin Ma

Department of Breast Surgery, Guangzhou Women and Children's Medical Center, 402 Renmin

Middle Road, Yuexiu District, Guangzhou City, China; Email: hongminmaa@sina.com; Tel:

$+8618902268759$

\#These authors contributed equally to this work. 


\section{Abstract}

Introduction: Plugged ducts and mastitis are common conditions in lactating women. Milk excretion and relief of local symptoms are equally important for the treatment of plugged ducts and mastitis. This study aimed to describe clinical response to 5-step systematic therapy (FSST) in the management of plugged ducts and mastitis. FSST was a comprehensive milk stasis dredging treatment, which contained five steps to make the milk out of the plugged duct.

Materials and Methods: This retrospective study included 922 breastfeeding women, 714 with plugged ducts and 208 with mastitis who received FSST at the breast nursing outpatient department from June to September 2017. The breast pain score, swelling degree and range of breast induration were recorded pre-FSST and post-FSST.

Results: After a single FSST, pain score and degree of swelling were significantly improved (both, $p<0.001$ ) in all cases. After receiving FSST, the mean breast pain relief score was $1.7 \pm$ 0.7, while the mean swelling fade-away degree was $1.6 \pm 0.6$. In subgroup analysis, pain score and degree of swelling were significantly improved (both, $p<0.001$ ) in the plugged ducts group and the mastitis group. The score of pain relief in the plugged ducts group was less than that in the mastitis group $(1.6 \pm 0.7$ vs. $1.9 \pm 0.7, t=-5.301, p=0.000)$, while improvement of swelling fade-away was greater in the plugged ducts group than the mastitis group $(1.7 \pm 0.6$ vs. $1.5 \pm 0.6$, $t=3.488, p=0.001)$. The composition ratio of the changes in induration range between the two groups was statistically different (Pearson $\chi^{2}=137.865, p=0.000$ ), of which more obvious improvement in the plugged ducts group than the mastitis group $\left(\chi^{2}=25.653, p=0.000\right)$.

Conclusion: FSST can relieve pain, reduce breast swelling and range of induration, and for 
plugged ducts or mastitis varied degree differently.

Keywords: Breastfeeding, breastfeeding support, massage, plugged ducts, breast pain, mastitis

\section{Introduction}

Because of benefits to both infants and mothers, breastfeeding is recommended by the World Health Organization (WHO) [1-4]. Furthermore, exclusive breastfeeding is recommended for infants from birth to 6 months [5]. Inflammatory breast diseases, such as plugged ducts and acute mastitis, are common conditions that afflict women during lactation, causing breast pain and discomfort. Studies have shown that breast inflammation during lactation is one of the most important iatrogenic causes of weaning [6,7]. Therefore, the efficient management of plugged breast ducts and acute mastitis during lactation is key to ensure successful breastfeeding [8]. According to WHO guidelines, plugged ducts and mastitis are different stages of breast inflammation during lactation caused by the blockage of lactiferous ducts as the initial factor [9]. There is no clear boundary between the two conditions, but mastitis is accompanied by more serious local and / or systemic inflammation manifestation. Significant fever, especially when associated with breast erythema and systemic symptoms such as myalgias, suggests the diagnosis of mastitis $[10,11]$. Both conditions may develop into a mammary abscess if they are not treated promptly and properly [12]. As such, effective treatment of plugged ducts and acute mastitis is particularly important in the management of lactation. The common pathogenesis of both plugged ducts and acute mastitis is inefficient milk emptying. It has been reported that timely and effective breast emptying is important to prevent and manage plugged ducts and acute mastitis $[8,9]$. 
A number of measures to stimulate efficient breast emptying have been explored, including massage, milk expression, breast pumping, cold and hot compression, acupuncture, auricular therapy, Gua-Sha therapy, ultrasonic treatment, and laser therapy [13-20]. When a single measure proves effective, combination of multiple measures should yield better results. Based on the aforementioned information, we combined five measures and developed a therapy called 5-step systematic therapy (FSST). The Breast Surgery Department of our hospital has been performing FSST for patients with inflammatory conditions during lactation since 2015. Until now, more than ten thousands patients received this therapy and achieved good results. We found the symptoms improvement of the patients varied by different inflammatory stage. Commonly used evaluation indicators include ratings of pain, swelling, hardness [21, 22]. The purpose of this study is to describe clinical response of lactating women with plugged ducts and / or acute mastitis treated with FSST from June to September 2017.

\section{Materials and Methods}

\section{Design}

This study was a retrospective analysis of lactating women with plugged ducts and / or acute mastitis women treated with FSST. The study was approved by the Ethics Committee of Guangzhou Women and Children`s Medical Center. Because of the retrospective nature of the study, the requirement of informed patient consent was waived.

\section{Setting}


This study was performed at Guangzhou Women and Children`s Medical Center, the largest Women and Children specialized hospital in south China. This hospital is a third-level grade A hospital, and is the first public hospital certified by the Joint Commission International (JCI) in

China. All pregnant women receive breastfeeding education according to the Chinese BabyFriendly Hospital Standards.

\section{Sample}

The study included a convenience sample of all lactating women who received FSST from June to September 2017. The symptoms of lactating women with plugged ducts and / or acute mastitis did not relieved after frequently breastfeeding for 48 hours. These women who refered to our department and received FSST were brought into our analysis. Exclusion criteria were patients who received FSST for other reasons, e.g., hypogalactia or breast abscess. Patients who did not complete treatment were also excluded. In fact, all patients should be evaluated and prescribed by a breast specialist prior to treatment. Suspected benign or malignant tumor should enter the corresponding treatment process, and were excluded from this study.

During the study period, 1,078 patients completed FSST. Of these, 156 (14.5\%) were excluded: 100 patients with hypogalactia and 56 with breast abscesses. A total of 922 patients met the criteria and were included in this study, and were divided into plugged ducts group (PDG, $\mathrm{n}=714$ ) and mastitis group (MG, $\mathrm{n}=208)$. 


\section{FSST}

FSST is performed by breast specialist outpatient nurses. The 5 steps of FSST are as follows.

1) Laser therapy. With the patient in the sitting position, the breast skin is fully exposed and irradiated by a SUNDOM-300 IB/233 LCD-type semiconductor laser therapy unit (Beijing SUNDOM Medical Equipment Co. Ltd., China). This Semiconductor laser instrument adopts multi-wavelength composite output $(650 / 810 \mathrm{~mm})$ which is suitable for various irradiation modes. The procedure of laser therapy is 1200 milliwattsof power for 10 minutes, irradiation area $8800 \mathrm{~mm}^{2}$, irradiation distance $10-15 \mathrm{~cm}$.If both breasts are affected, both should be irradiated at the same time. The nipple/areola is covered with gauze during irradiation.

2) Electric breast pumping. A Medela Swing medical breast pump(Medela AG, Switzerland) was used for bilateral breast pumping. Beginning at zero, the negative pressure was increased until the patient's maximum discomfort level was reached. Pumping was continued for 10-15 minutes.

3) Breast massage. Patients were placed in the supine position with 2 dry towels under their body. After the comprehensive evaluation of both breasts, the massage was started from the contralateral breast. The root of the nipple was softly massaged for 30 seconds for the milk ejection reflex. The areola was pressed from different directions to open the milk ducts. The breast was pushed and kneaded from the base toward the areola to excrete the milk, and then the nipple and areola were squeezed again to discharge the milk. Hard knots were gently massaged with the thumb or hypothenar muscles until softened. Unilateral breast massage was performed for no more than 15 minutes. 
4) Magnesium sulfate application. Gauze soaked in a 33\% magnesium sulfate solution was applied to both sides of the breast for 10-15 minutes.

5) Patient education. Patients were given individualized guidance for breastfeeding based on their existing problems. The benefits of breastfeeding were reinforced, and they were encouraged to increase their self-confidence for breastfeeding.

\section{Outcome measures}

Pain score, degree of breast swelling, and range of induration were recorded before and after treatment. Pain was graded on a numerical rating scale: 0 points for no pain; 1-3 points for mild pain, 4-6 points for moderate pain, and 7-10 points for severe pain. The pain score before treatment minus that after treatment was defined as the pain relief score; the higher the score, the greater the pain relief.

The degree of swelling was assessed using the scale developed by Humenick et al [23]. The scale is as follows: 1 degree, the breast is soft, no changes; 2 degrees, slight breast swelling; 3 degrees, breast swelling is present, no pain (0 points); 4 degrees, breast distension, mild pain (13 points); 5 degrees, breast distension, moderate pain (4-6 points); 6 degrees, the breast is very swollen and there is severe pain (7-10 points). Swelling degree was recorded before and after treatment. The swelling degree before treatment minus that after treatment was defined as the swelling fade-away degree; the higher the score, the greater the improvement.

The range of induration was recorded before and after treatment. The changes in the range of induration were recorded by modified quantitative grades [22]. The grades are as follows: 
Disappearance (2) : the range of the hard lump was reduced by more than $80 \%$ after treatment;Reduction (1) : the range was reduced by $10 \%-80 \%$ after treatment;No change (0) : the range was between $-10 \%$ and $10 \%$ after treatment; Aggravation $(-1)$ : the range increased by more than $10 \%$ after treatment; Unable to evaluate (-). The higher the score, the greater the improvement.

\section{Data collection}

Data was collected from June to September 2017 by the breast specialist outpatient nurses who performed the treatments. To ensure accuracy and consistency of the data collection process, a training session was provided to all the breast specialist outpatient nurses involved in this study. Patient demographic information and the time of onset of the condition were recorded before treatment, and pain score, swelling degree, and range of induration were recorded before and after treatment. Data were entered and stored into IBM Statistical Package for the Social Sciences 22.0 (SPSS) software for analysis (IBM Corp, 2013) immediately after collection. All participants were assured that all data would be kept secure, and participation was completely voluntary. All data obtained was used only by the researchers.

\section{Data analysis}

Descriptive statistics including median, range, and frequency were calculated. Items were first summed to create a score for age, onset time, pain level, swelling degree, changes in the range of induration. Single sample t-test, 2-independent-samples test and Pearson Chi-square were used 
to assess the variables. For all analyses, a value of $p<0.050$ was considered to indicate statistical significance.

\section{Results}

\section{Patient basic information}

The mean age of all patients was $30.4 \pm 3.9$ years. The oldest patient was 43 years, and the youngest was 17 years. The most common onset time of all patients was during the first to the third month postpartum, accounting for $41.6 \%$, and the incidence rate within 3 month postpartum was as high as $80.7 \%$. According to PDG and MG, characteristics of the 2 groups are summarized in Table 1.

\section{Pain}

The overall mean pain score before treatment was $3.8 \pm 1.5$, with a maximum of 7.0 and a minimum of 0.0 . The mean pain score pre-FSST in the plugged ducts group was $3.2 \pm 1.2$, with a maximum of 7.0 and a minimum of 0.0 . In the MG, the mean pain score pre-FSST was $5.7 \pm$ 0.7, with a maximum of 7.0 and a minimum of 4.0 . The pain score pre-FSST of the MG was higher than that of the PDG, and the difference was statistically significant $(t=-28.126, p=$ $0.000)$.

The overall mean breast pain relief score was $1.7 \pm 0.7$. All patients had statistically significant difference in pain relief score after treatment $(t=73.542, p=0.000)$. In the PDG, the mean pain 
relief score was $1.6 \pm 0.7$, with a maximum pain relief score of 4.0. No patient experienced pain aggravation after treatment, but some patients did not experience relief of symptoms. In the MG, the mean pain relief score was $1.9 \pm 0.7$, and the maximum pain relief score was 3.0. One patient experienced aggravation of pain after treatment, with a score of -1 . There was a statistically significant difference in pain relief score between the 2 groups $(t=-5.301, p=0.000)$, with greater pain relief in the MG (Figure 1).

\section{Swelling}

The mean swelling degree of all patients before treatment was $3.9 \pm 1.2$, with a maximum of 6.0 and a minimum of 1.0. The mean swelling degree pre-FSST in the PDG was $3.5 \pm 1.1$, with a

maximum of 6.0 and a minimum of 1.0. The mean swelling degree pre-FSST in the MG was 5.2 \pm 0.6 , with a maximum of 6.0 and a minimum of 4.0 . The swelling degree pre-FSST of the MG was higher than that of the PDG, and the difference between the 2 groups was statistically significant $(t=-21.658, p=0.000)$.

In the whole population, the mean swelling fade-away degree was $1.6 \pm 0.6$. All patients had statistically significant difference in swelling fade-away degree after treatment $(t=78.464, p=$ 0.000 ). In the PDG, the mean swelling fade-away degree was $1.7 \pm 0.6$, with a maximum of 3.0 , and with a minimum of 0.0 . In the MG, the mean swelling fade-away degree was $1.5 \pm 0.6$, with a maximum of 3.0, and with a minimum of 0.0. There was a statistically significant difference in the swelling fade-away degree between the 2 groups ( $t=3.488, p=0.001$ ), with the swelling fade- 
away greater in patients with plugged ducts (Figure 2).

\section{Range of breast induration}

All breast induration in all patients could be evaluated. Based on the scoring system, in the overall population the frequency of a score of 2 (disappearance) was $31.7 \%(292 / 922), 1$ (reduction) was 63.9\% (589/922), 0 (no change) was 4.4\% (41/922), and -1 (aggravation) was 0 (0/922). In the PDG, the frequency of a score of 2 was $40.9 \%(292 / 714), 1$ was $56.6 \%$ (404/714), and 0 was $2.5 \%(18 / 714)$, and the effective rate of treatment was $97.5 \%$. In the MG, the frequency of a score of 2 was 0,1 was $88.9 \%(185 / 208)$, and 0 was $11.1 \%(23 / 208)$, and the effective rate of treatment was $88.9 \%$ (Figure 3 ). There was a statistically significant difference in the composition ratio of the changes in induration range between the two groups

(Pearson $\left.\chi^{2}=137.865, p=0.000\right)$. According to the effective rate of treatment (the frequency of 1 and 2), the range of breast induration was improved more obviously in the PDG than the MG $\left(\chi^{2}=25.653, p=0.000\right)$

\section{Discussion}

Plugged ducts, acute mastitis, mammary abscesses are collectively called inflammatory breast diseases during lactation. Plugged ducts often occurs 2-3 weeks after delivery [24], because of injury to the nipple, ineffective milk removal, poor breastfeeding technique, irregular lactation, superficial pressure on the breast, and an overabundant milk supply [9, 25]. Because of delayed resolution, plugged ducts may lead to more severe symptoms, such as mastitis and mammary 
abscess [24]. Acute mastitis can happen at any time during lactation, but is mostly seen in the first 6 weeks postpartum $[8,26]$. Whatever plugged ducts or mastitis, the discomfort and pain can affect the mother's confidence in breastfeeding, cause anxiety, and can lead to early weaning [27]. In our study, plugged ducts occurred more frequently within 1 month of postpartum, while mastitis occurred in 1-3 months of postpartum. No matter the onset time of plugged ducts or mastitis was similar in previous study.

Plugged ducts and mastitis can be effectively relieved with sufficient breast emptying. A baby feeding is the most effective way to empty the breast. However, because of the separation of maternal and infant diseases, nipple injury, congenital malformation, or the blocked milk tube hard to be recanalization by infant sucking, some mothers have to seek medical help. Our hospital developed a comprehensive milk stasis treatment called FSST, which can effectively increase stasis milk excretion to relieve the symptoms of patients with plugged ducts and mastitis. Previous studies have used similar comprehensive treatments, and obtained similar positive results [28].

In our study, the symptoms of the patients, including pain and swelling, were significantly improved after receiving FSST, which to some extent indicated that the FSST scheme had certain curative effect on these patients. Previous studies have shown that the clinical symptoms at different stages of lactation can be effectively alleviated after treatment [29]. But there is no prior data to show whether the clinical response on different symptoms varies at different stages after effective treatment. We focused on the clinical response to FSST on patients with plugged ducts and those with mastitis, and an interesting phenomenon was found. FSST provided better 
pain relief in the MG, whereas breast swelling fade-away and reduction of hard lump was greater in the PDG. Breast swelling fade-away and hard lumps reduction both reflected the objective situation of the disease change, indicating the degree of milk excretion, the results of both should be consistent, and statistical analysis also supported this conclusion. In theory, the breast swelling fade-away and the lumps reduction was better in the PDG, suggesting greater pain relief, but the opposite was observed. There may be 2 reasons for this finding. The pretreatment pain score of the MG was higher than that of the plugged ducts group. The higher the score, the more obvious it falls. Patients had a strong feeling about the improvement of pain. Second, breast pain is not only caused by the mechanical expansion of milk ducts caused by plugged ducts, but also by an increase in inflammatory factors such as prostaglandins, bradykinin, and substance P [30]. In the animal studies, extracellular matrix (ECM) increases during mastitis stage, and the increased ECM such as prostaglandins can cause more serious pain $[31,32]$. Human study has shown there are considerable differences in the concentrations of many components of human milk before and after breast massage [33]. FSST can stimulate stasis milk excretion, and milk excretion may affect the concentration of ECM. We hypothesized that pain relief in mastitis after FSST is on the one hand due to the physical effect of stasis milk excretion, on the other hand due to the chemical effect of milk excretion causing a decrease in the concentration of pain-related factors. Further research is needed to confirm the hypothesis. Changes in induration range could be observed that the lumps could vanish completely in quite a number of patients in PDG (40.9\%) after treatment, but none of the patients in mastitis group, means that the number of mastitis patients might need to accept more treatment (more times 
FSST, medicine and so on) to achieve a better therapeutic effect.

Since 2015, more than 10,000 patients have been treated with FSST at our hospital. In fact, some patients were referred to our hospital for further diagnosis and treatment due to poor therapeutic effects. The safety and effectiveness of FSST is based on the cooperation of medical and nursing care, and full evaluation of the patient's condition. Therefore, it is not recommended to promote it on the basis of no standardized diagnosis and treatment process in China. There are various forms of breast massage in the society at present. It also gives birth to a new profession called "Cui Ru Shi" in China. However, due to the lack of standardized training and professional medical knowledge, "Cui Ru Shi" not only can not alleviate the pain of patients, but also aggravate the disease [34].

There are some shortcomings in the study. FSST is a complex therapeutic protocol, and the most effective scheme should be explored. In addition, the difference of clinical response to FSST between the two groups is still in observation research. There is lack of pathophysiological evidence, such as the hypothesis that treatment can reduce the concentration of pain-related factors in ECM, and the analysis of milk is still to be further studied in the next stage.

\section{Conclusion}

FSST relieved breast pain, reduce breast swelling and range of induration. An interesting phenomenon was found that clinical response was different between two groups. However, the FSST scheme requires optimized, and the mechanisms of difference in clinical response need to be further studied. 


\begin{abstract}
Abbreviations
FSST: 5-step systematic therapy; WHO: World Health Organization; JCI: Joint Commission International; PDG: plugged ducts group; MG: mastitis group; ECM: extracellular matrix.
\end{abstract}

\title{
Acknowledgments
}

We would like to give our special thanks to our patients who participated in the project.

\section{Authors' contributions}

Y.Y.Z. and L.T.Z. both collected data, performed the data analysis, and wrote the manuscript.

M.H.M. conceived the study idea and made suggestions for revision. Y.Y.Z., P.Y.H., L.Y., W.L. and F.B.J. performed FSST and collected data. All authors read and approved the final manuscript.

\section{Funding}

The authors disclosed no receipt of the financial support for the research, authorship, and/or publication of this article.

\section{Availability of data and materials}

"The datasets used and/or analyzed during the current study are available from the 
corresponding author on reasonable request” .

\title{
Ethics approval and consent to participate
}

The study was approved by the Ethics Committee of Guangzhou Women and Children`s

Medical Center. Because of the retrospective nature of the study, the requirement of informed patient consent was waived.

\section{Consent for publication}

Not applicable.

\section{Competing interests}

The authors declared no potential conflicts of interest with respect to the research, authorship, and/or publication of this article.

\author{
Author details \\ Department of Breast Surgery, Guangzhou Women and Children's Medical Center, 402 \\ Renmin Middle Road, Yuexiu District, Guangzhou City, China.
}

\section{References}

1. Jeurink P V , Van B J , Jiménez $E$, et al. Human milk: a source of more life than we imagine[J]. Beneficial Microbes, 2013, 4(1):17-30. 
2. American Academy of Paediatrics (AAP), 2012. Breastfeeding and the use of human milk. Pediatrics 129: e827-841.

3. Li D P , Du C , Zhang Z M , et al. Breastfeeding and ovarian cancer risk: a systematic review and meta-analysis of 40 epidemiological studies[J]. Asian Pacific Journal of Cancer Prevention Apjcp, 2014, 15(12):4829.

4. Yang Z, Lai J, Yu D , et al. Breastfeeding rates in China: a cross-sectional survey and estimate of benefits of improvement[J]. The Lancet, 2016, 388:S47.

5. Jama A, Gebreyesus H, Wubayehu T, et al. Exclusive breastfeeding for the first six months of life and its associated factors among children age 6-24 months in Burao district, Somaliland[J]. International Breastfeeding Journal, 2020, 15(1):5.

6. Schwartz K, D'Arcy H J, Gillespie B, et al. Factors associated with weaning in the first 3 months postpartum[J]. Journal of Family Practice, 2002, 51(5):439.

7. Mangesi $L$, Dowswell $T$. Treatments for breast engorgement during lactation[J]. Cochrane Database Syst Rev, 2010, 65(9):CD006946.

8. Amir L. ABM clinical protocol \#4: mastitis[J]. Breastfeed Med. 2014, 9(5):239-243.

9. World Health Organization. Mastitis: Causes and Management[M]. Geneva, Switzerland: World Health Organization, 2000.

10. Pamela B , Wendy B . ABM Clinical Protocol \#20: Engorgement, Revised 2016[J]. Breastfeed Med, 2016, 05(11):159-163.

11. Contreras G A, Juan Miguel Rodrí guez. Mastitis: Comparative Etiology and Epidemiology[J]. J Mammary Gland Biol Neoplasia, 2011, 16(4):339-356. 
12. Prachniak, Gail K . Common breastfeeding problems[J]. Obstetrics and gynecology clinics of North America, 2002, 29(1):77-88.

13. Ayers J F . The use of alternative therapies in the support of breastfeeding[J]. J Hum Lact, 2000, 16(1):52-56.

14. Ryukhova I . History of Breastfeeding in Russia[M]. Moscow, Russia: Eksmo, 2010:10-20.

15. Chiu J Y, Gau M L, Kuo S Y, et al. Effects of Gua-Sha therapy on breast engorgement: a randomized controlled trial[J]. J Nurs Res, 2010, 18(1):1-10.

16. Sukhee $A$, Jinhee $K$, Jungsuk $C$. Effects of Breast Massage on Breast Pain, Breast-milk Sodium, and Newborn Suckling in Early Postpartum Mothers[J]. Journal of Korean Academy of Nursing, 2011, 41(4):451-459.

17. Lavigne V , Gleberzon B J . Ultrasound as a treatment of mammary blocked duct among 25 postpartum lactating women: a retrospective case series[J]. J Chiropr Med, 2012, 11(3):170178.

18. Bolman M , Saju L , Oganesyan K, et al. Recapturing the Art of Therapeutic Breast Massage during Breastfeeding[J]. J Hum Lact, 2013, 29(3):328-331.

19. Miranda L, Eckereder, Amir, et al. Low level laser therapy for breastfeeding problems[J]. Breastfeeding Review Professional Publication of the Australian Breastfeeding Association, 2016, 24(2):27-31.

20. Chen L S, Tan J Y, Suen K P. Auricular therapy for lactation: A systematic review[J]. Complementary Therapies in Clinical Practice, 2017:S1744388117302232.

21. Mangesi L, Dowswell T. Treatments for breast engorgement during lactation[J]. Cochrane 
Database Syst Rev, 2010, 65(9):CD006946.

22. Anderson L, Kynoch $\mathrm{K}$, Kildea S. Effectiveness of breast massage in the treatment of women with breastfeeding problems[J]. JBI Database of Systematic Reviews and Implementation Reports, 2016, 14(8):19-25.

23. Humenick S S, Hill P D, Anderson M A . Breast Engorgement: Patterns and Selected Outcomes[J]. J Hum Lact, 1994, 10(2):87-93.

24. Meng S, Deng Q, Feng C, et al. Effects of massage treatment combined with topical cactus and aloe on puerperal milk stasis[J]. Breast Disease, 2015, 35(3):173-178.

25. Abou-Dakn M, Richardt A, Schaefer-Graf U, et al. Inflammatory Breast Diseases during Lactation: Milk Stasis, Puerperal Mastitis, Abscesses of the Breast, and Malignant Tumors Current and Evidence-Based Strategies for Diagnosis and Therapy[J]. Breast Care, 2010, $5(1): 33-37$.

26. Egbe $\mathrm{T} \mathrm{O}$, Njamen $\mathrm{T} \mathrm{N}$, Essome $\mathrm{H} \mathrm{T}$, et al. The estimated incidence of lactational breast abscess and description of its management by percutaneous aspiration at the Douala General Hospital, Cameroon[J]. International Breastfeeding Journal, 2020, 15(1):26.

27. Kabir S, Tasnim S. Oketani lactation management: a new method to augment breast milk[J]. J Bangladesh Coll Physicians Surg, 2009, 27(3):155-159.

28. Zhao C, Tang R, Wang J , et al. Six-step recanalization manual therapy: a novel method for treating plugged ducts in lactating women[J]. J Hum Lact, 2014, 30(3):324-330.

29. Witt A M, Bolman M, Kredit S, et al. Therapeutic Breast Massage in Lactation for the Management of Engorgement, Plugged Ducts, and Mastitis[J]. J Hum Lact, 2016, 32(1):123-131. 
30. Deist F L, De Saint-Basile G, Angeles-Cano E, et al. Prostaglandin E2 and plasminogen activators in human milk and their secretion by milk macrophages[J]. American Journal of Reproductive Immunology and Microbiology, 1986, 11(1):6-10.

31. Bulgari $O$, Dong X, Alfred L, et al. Innate immune responses induced by lipopolysaccharide and lipoteichoic acid in primary goat mammary epithelial cells[J]. Journal of Animal Science \& Biotechnology, 2017, 8:29.

32. Hockett $M E$, Hopkins $F M$, Lewis $M J$, et al. Endocrine profiles of dairy cows following experimental induced clinical mastitis during early lactation[J]. Animal Reproduction Science, 2000, 58(3-4):241-251.

33. Mervat I, Kawashima $T$, Nakamura $S$, et al. Composition of milk obtained from unmassaged versus massaged breasts of lactating mothers[J]. Journal of Pediatric Gastroenterology and Nutrition, 2004, 38(6):484-487.

34. Eidelman A I. The Appropriate Use of Breast Massage[J]. Breastfeed Med, 2016, 11(9):423. 


\section{Figure legends}

Figure 1. Clustered Error bar graph of pain score by separated symbols in ALL, PDG and MG.

*Compared with PDG, the difference was statistically significant in MG $(p<0.050)$.

Figure 2. Clustered Error bar graph of swelling degree by separated symbols in ALL, PDG and MG.

*Compared with PDG, the difference was statistically significant in MG $(p<0.050)$.

Figure 3. Pie charts of change score in induration range.

(A) Change score in induration range of the whole population. (B) Change score in induration range of the PDG. (C) Change score in induration range of the MG. (D) Line chart of change score in induration range of the whole population and the 2 groups. $(2$ : disappearance; 1 :

reduction; 0 : no change) 
Figures

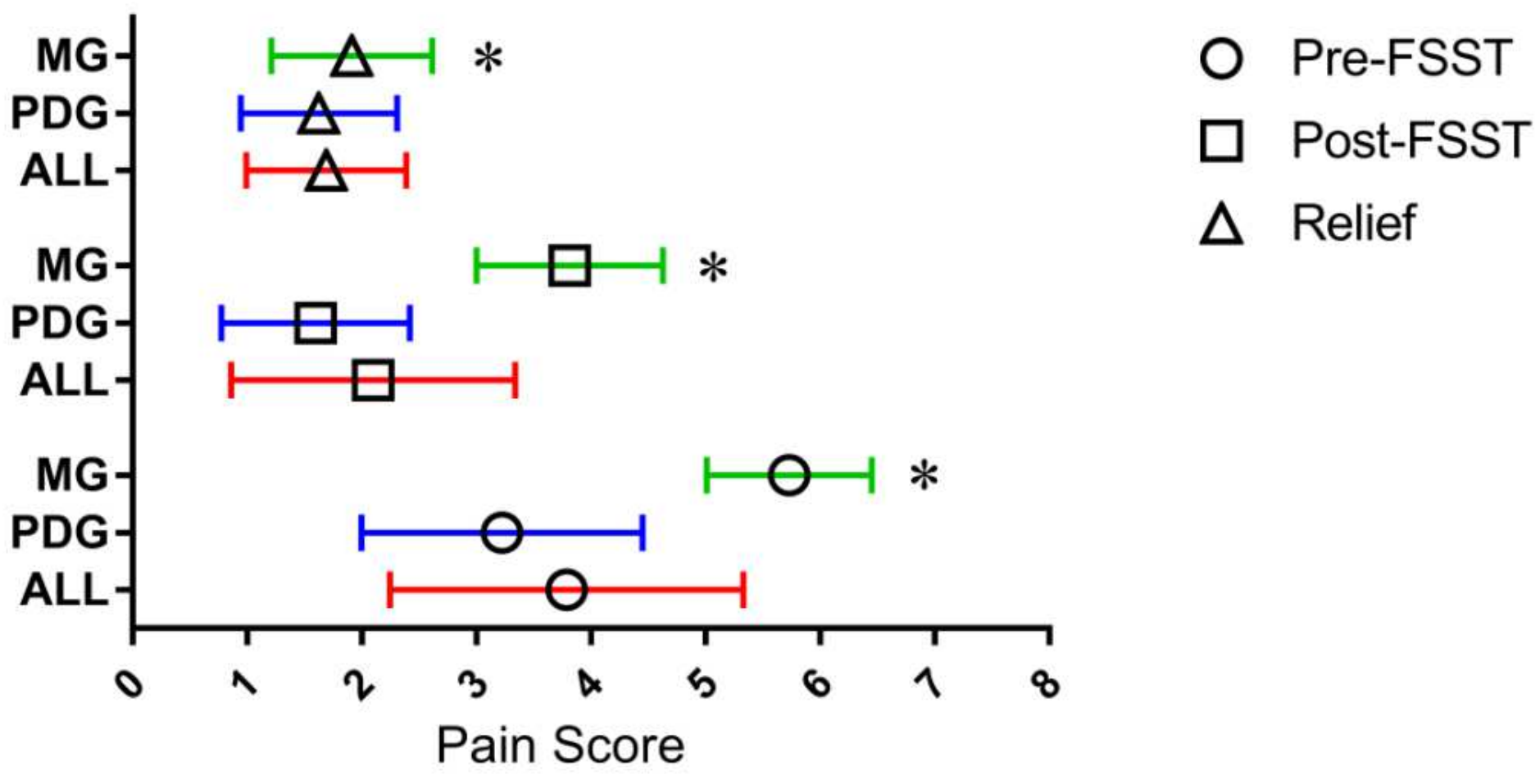

Figure 1

Clustered Error bar graph of pain score by separated symbols in ALL, PDG and MG. *Compared with PDG, the difference was statistically significant in MG $(p<0.050)$. 


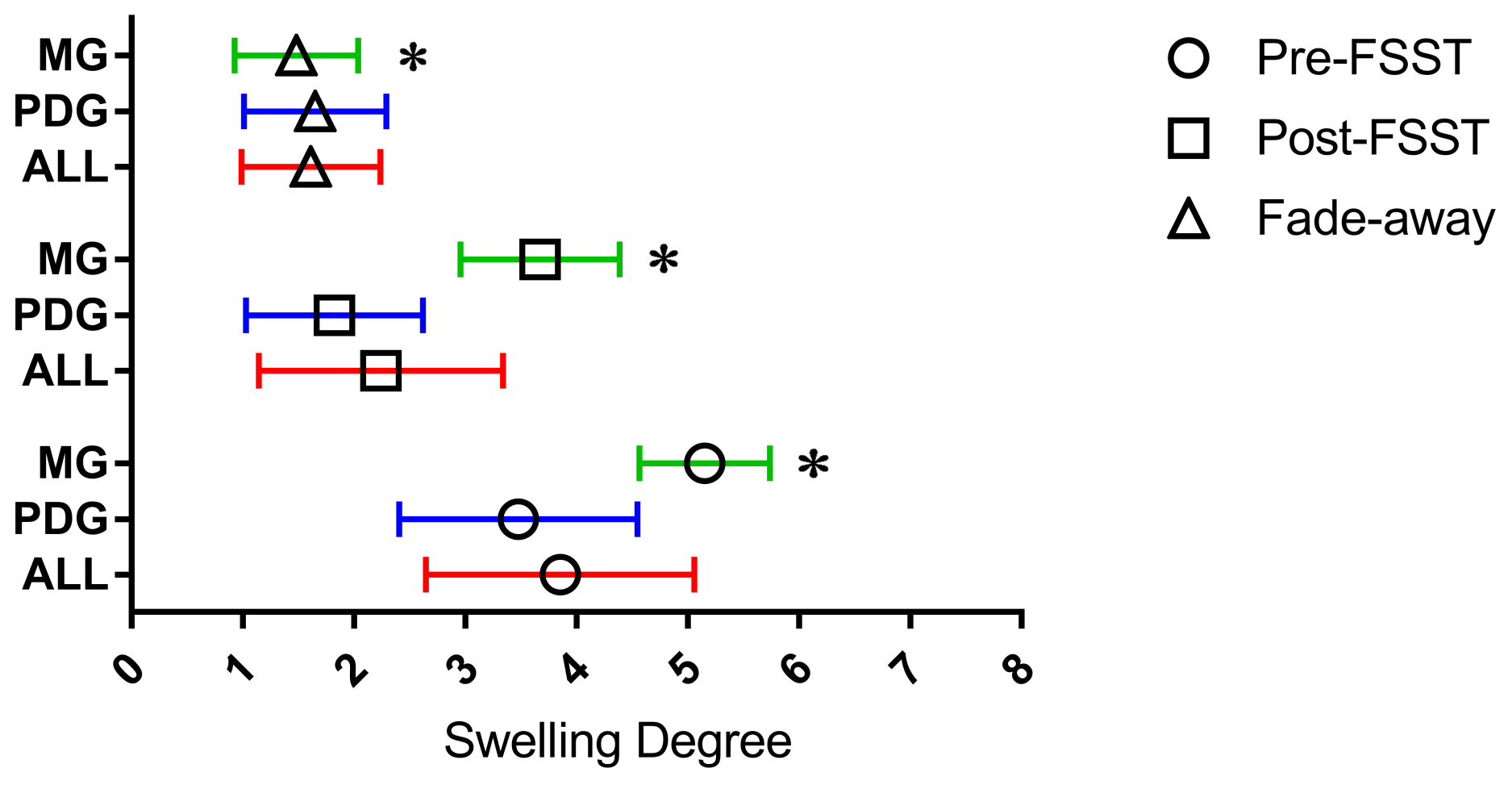

Figure 2

Clustered Error bar graph of swelling degree by separated symbols in ALL, PDG and MG. *Compared with PDG, the difference was statistically significant in MG $(p<0.050)$. 
A

ALL

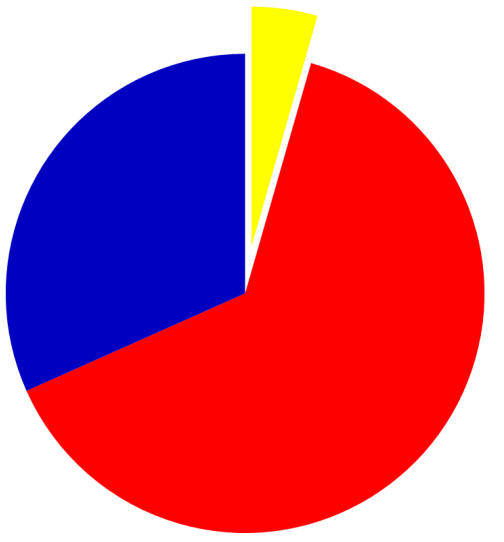

Total $=922$

C

MG
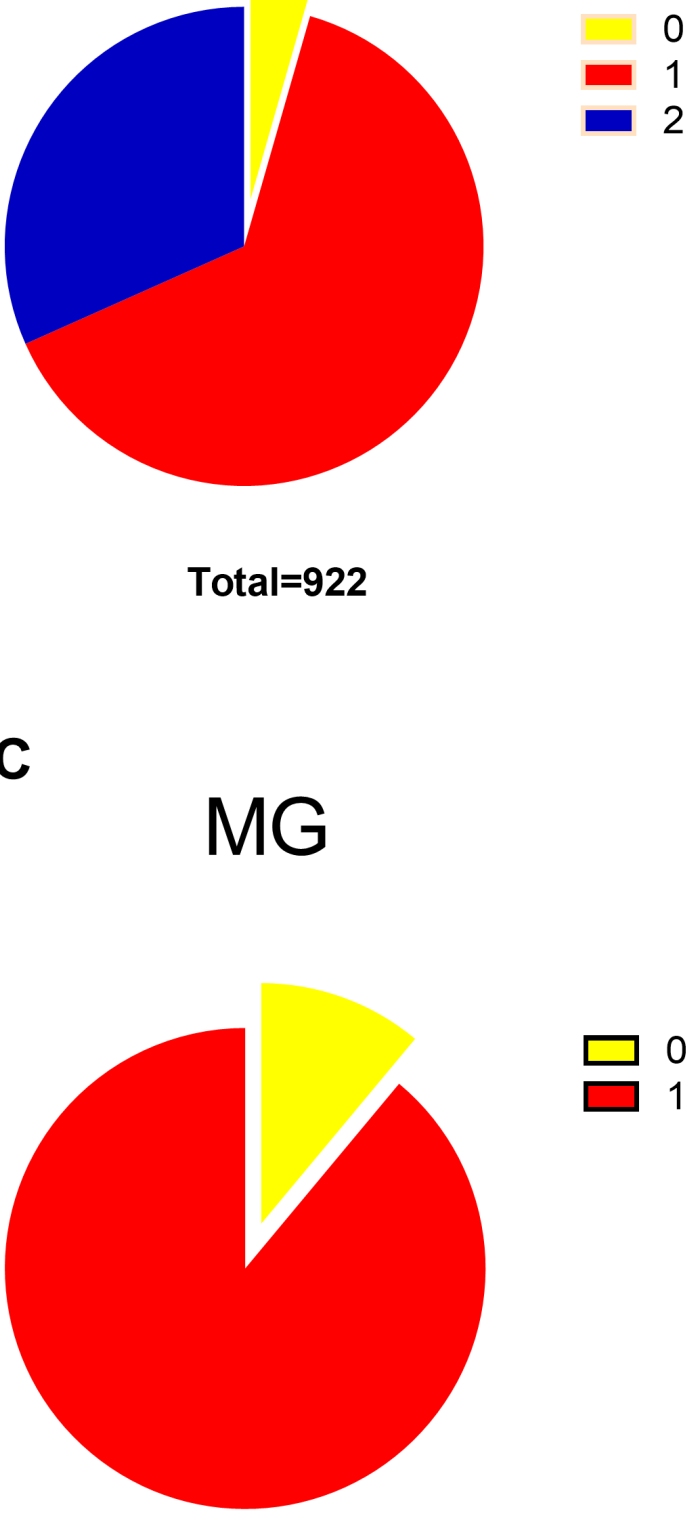

Total $=\mathbf{2 0 8}$
B

\section{PDG}

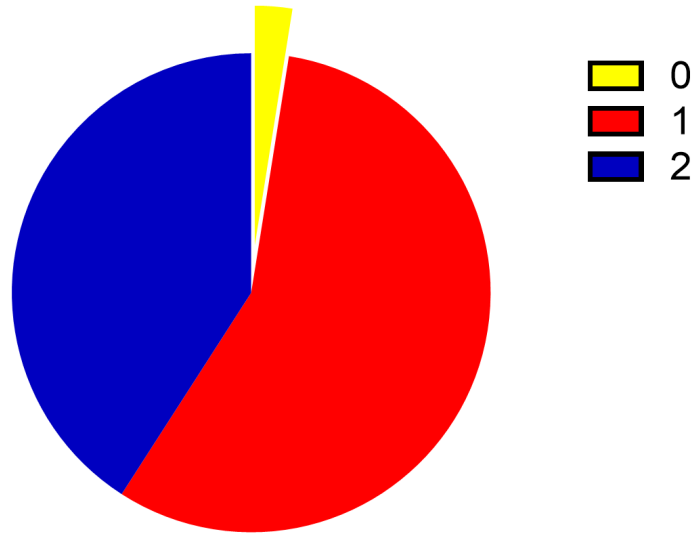

Total=714

D

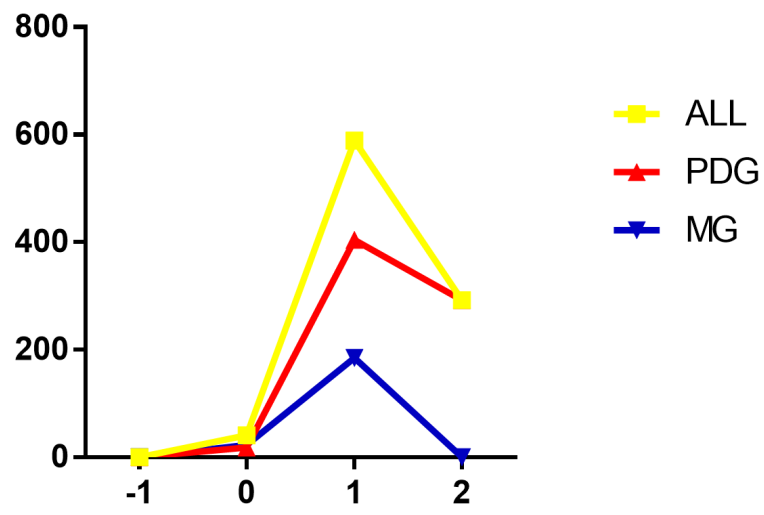

\section{Figure 3}

Pie charts of change score in induration range. (A) Change score in induration range of the whole population. (B) Change score in induration range of the PDG. (C) Change score in induration range of the MG. (D) Line chart of change score in induration range of the whole population and the 2 groups. (2: disappearance; 1 : reduction; 0 : no change) 